\title{
The central nucleus of the amygdala is essential for acquiring and expressing conditional fear after overtraining
}

\author{
Joshua M. Zimmerman, ${ }^{1,2}$ Christine A. Rabinak, ${ }^{1}$ Ian G. McLachlan, ${ }^{1}$ \\ and Stephen Maren ${ }^{1,2,3}$ \\ ${ }^{1}$ Department of Psychology, University of Michigan, Ann Arbor, Michigan 48109-1043, USA; ${ }^{2}$ Neuroscience Program, University \\ of Michigan, Ann Arbor, Michigan 48109-1043, USA
}

\begin{abstract}
The basolateral complex of the amygdala (BLA) is critical for the acquisition and expression of Pavlovian fear conditioning in rats. Nonetheless, rats with neurotoxic BLA lesions can acquire conditional fear after overtraining (75 trials). The capacity of rats with BLA lesions to acquire fear memory may be mediated by the central nucleus of the amygdala (CEA). To examine this issue, we examined the influence of neurotoxic CEA lesions or reversible inactivation of the CEA on the acquisition and expression of conditional freezing after overtraining in rats. Rats with pretraining CEA lesions (whether alone or in combination with BLA lesions) did not acquire conditional freezing to either the conditioning context or an auditory conditional stimulus after extensive overtraining. Similarly, post-training lesions of the CEA or BLA prevented the expression of overtrained fear. Lastly, muscimol infusions into the CEA prevented both the acquisition and the expression of overtrained fear, demonstrating that the effects of CEA lesions are not likely due to the destruction of en passant axons. These results suggest that the CEA is essential for conditional freezing after Pavlovian fear conditioning. Moreover, overtraining may engage a compensatory fear conditioning circuit involving the CEA in animals with damage to the BLA.
\end{abstract}

Pavlovian fear conditioning is an important model for studying the neural mechanisms contributing to emotional learning and memory (Davis 1992; LeDoux 2000; Maren 2001, 2005a). In this paradigm, a conditioned stimulus (CS), such as a tone, is presented with an aversive unconditional stimulus (US), such as a footshock. The pairing of the CS and the US comes to elicit conditioned fear responses (CRs), including increased heart rate, blood pressure, acoustic startle, and somatomotor immobility (i.e., freezing). It is now well established that the amygdala is critical for this form of learning (Fendt and Fanselow 1999; LeDoux 2000; Davis and Whalen 2001; Maren 2001). The majority of current work focuses on the role of the nuclei within the amygdala, specifically the basolateral complex (BLA: consisting of the lateral [LA], basolateral [BL], and basomedial [BM] nuclei) and the central nucleus (CEA), in this form of learning. Within the amygdala, the BLA is believed to be the site at which information regarding the CS (auditory and contextual cues) and the US first converge, although CEA neurons also receive auditory and somatic input. Afferents from the medial geniculate body (MGm) (Doron and Ledoux 2000), as well as various sensory cortices including the primary auditory cortex (Romanski and LeDoux 1992), route information regarding the CS to the LA. Afferents from the hippocampus transmit multimodal information regarding the context and time of conditioning to the BLA (O'Reilly and Rudy 2001; Sanders et al. 2003); highly processed sensory information from cortical regions, including the prefrontal cortex (PFC), also converges in the LA (McDonald 1998).

In contrast, the medial division of the CEA (CEm) has been posited to be the primary output structure of the amygdala. The

${ }^{3}$ Corresponding author.

E-mail maren@umich.edu; fax (734) 763-7480.

Article is online at http://www.learnmem.org/cgi/doi/10.1101/lm.607207.
CEA receives information from the LA via the intercalated nuclei, and it also receives direct projections from the BL and thalamus. The CEm, in turn, projects to brain areas involved in the production of the CR, including the periaqueductal gray and the lateral hypothalamus, which mediate freezing and cardiovascular response, respectively (LeDoux et al. 1988). However, recent studies suggest that the CEA may also have a role in the acquisition of conditional fear (Goosens and Maren 2003; Maren 2005a; Wilensky et al. 2006), and it is anatomically positioned to serve this role (Pare et al. 2004). These findings lend support to two competing models of information processing within the amygdala during learning. In the serial model, information about the CS and US enter and are associated within the BLA, and this information is then transmitted to the CEA for the expression of fear. Alternatively, the parallel model proposes that the BLA and CEA both perform associative functions (for review, see Pare et al. 2004; Maren 2005b; Balleine and Killcross 2006), suggesting that one nucleus might compensate for the loss of the other under certain conditions.

Lesions of either the BLA or the CEA produce deficits in both the acquisition and the expression of conditional fear (LeDoux et al. 1990; Helmstetter 1992; Campeau and Davis 1995; Maren et al. 1996; Cousens and Otto 1998; Goosens and Maren 2001). However, despite previous findings that overtraining (25 CS-US trials) does not mitigate the effects of excitotoxic BLA lesions (Maren 1998), rats with BLA lesions can acquire conditional freezing after extensive overtraining (75 CS-US trials) (Maren 1999; Goosens and Maren 2003). Given the important role for the CEA in fear conditioning, it is possible that CEA neurons are involved in the acquisition and expression of conditional freezing in rats without an intact BLA. The current experiments address this possibility and reveal an essential role for the CEA in the acquisition and expression of conditional fear after extensive overtraining. 


\section{Results}

\section{Experiment 1: Neurotoxic CEA lesions prevent the acquisition of overtrained fear}

Rats with BLA lesions acquire conditional freezing after extensive overtraining in either contextual or auditory fear conditioning paradigms (Maren 1999). To determine the involvement of the CEA in conditional freezing after overtraining, we explored the effect of pretraining lesions of the BLA, CEA, or combined lesions of both the BLA and CEA on conditional freezing after a 75-trial auditory fear conditioning procedure. Short-term fear responses were assessed by measuring conditional freezing during the overtraining session. Long-term fear memory was assessed by independently measuring conditional freezing to the conditioning context and the auditory CS, which was presented in a novel context.

\section{Histology}

On the basis of the histological results, 19 of 74 rats were excluded. Rats were excluded if their lesions were larger than intended, misplaced, or largely unilateral. This yielded the following group sizes: CEA $(n=14)$, BLA $(n=8)$, CEA + BLA $(n=19)$, and $\mathrm{SH}(n=15)$. The extent of the amygdala damage for rats included in the analyses is depicted in Figure 1. As can be seen, damage was generally confined to the targeted nucleus. For lesions targeting the BLA, there was some damage to the rostral entorhinal cortex. For lesions targeting the CEA, there was minor damage to the caudate putamen and substantia innominata. Not surprisingly, the combined lesions of the CEA and BLA were more extensive than the individual CEA or BLA lesions.

\section{Behavior}

Post-shock freezing during the conditioning session is shown in Figure 2A. The data were analyzed using a two-way ANOVA with variables of lesion (SH, CEA, BLA, and CEA + BLA) and trial (15 5 -trial blocks). During the pretrial period rats displayed minimal levels of freezing $(<5 \%)$ before footshock. After the onset of conditioning, rats exhibited robust freezing. The ANOVA revealed a main effect of lesion $\left(F_{(3,47)}=19.5 ; P<0.0001\right)$ and a main effect

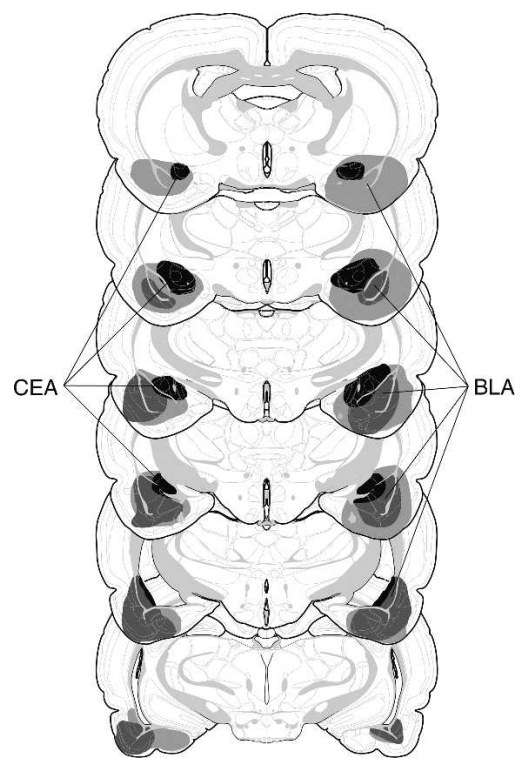

Figure 1. Schematic representation of the extent of pretraining NMDA lesions (median lesion) of the CEA (black), BLA (dark gray), and CEA and BLA lesions (light gray) for Experiment 1. Coronal brain section images adapted from Swanson (1992).
A

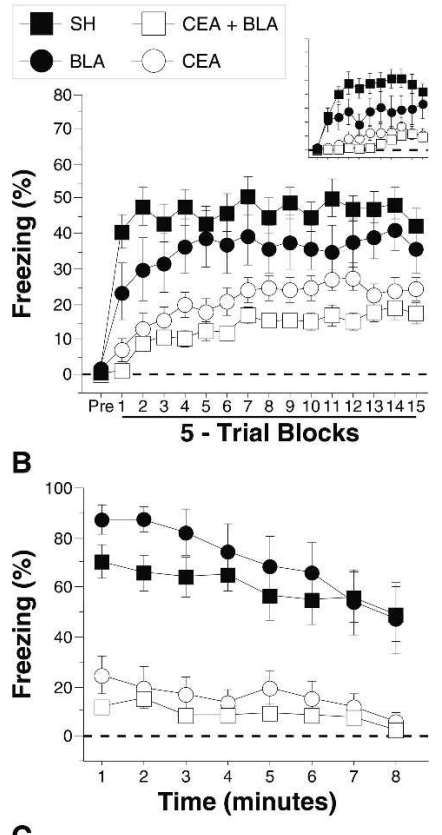

C

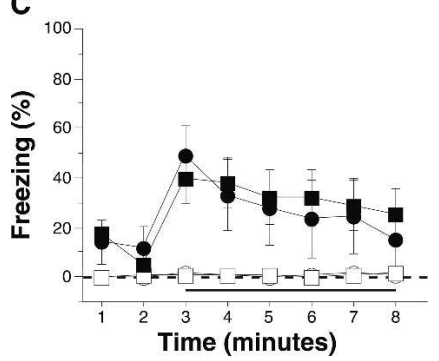

Figure 2. Conditioned freezing in rats with pretraining amygdala lesions (Experiment 1). (A) Mean percentage of freezing ( \pm SEM) during the 75 trial training session (data are displayed with a $30-\mathrm{sec}$ pretrial period followed by 15 bins consisting of 5 trials each). Freezing was quantified before the first conditioning trial (Pre) and during the 1-min period after each conditioning trial; these values were averaged in 5-trial blocks. The inset shows the mean percentage of freezing ( \pm SEM) during the preperiod through the first two training blocks expanded to show minutes $1-10$. (B) Mean percentage of freezing ( \pm SEM) to contextual (8-min context extinction test) cues $24 \mathrm{~h}$ after training. (C) Mean percentage of freezing ( \pm SEM) to the auditory CS in a novel context $48 \mathrm{~h}$ after training. The auditory CS was initiated $2 \mathrm{~min}$ after rats were placed in the chambers (horizontal bar indicates the CS). Data are shown for rats with lesions of the BLA (solid circles), CEA (open circles), or CEA + BLA (open squares); $\mathrm{SH}$ rats (solid squares).

of training trial $\left(F_{(14,658)}=10.4 ; P<0.0001\right)$ without a significant interaction of lesions $\times$ training trial $\left(F_{(42,658)}=1.2 ; P=0.22\right)$. This indicates that freezing differed among the groups across the training session. Post-hoc analysis of the main effect of lesion revealed a difference between $\mathrm{SH}$ rats and rats with either CEA lesions $(P<0.0001)$ or CEA + BLA lesions $(P<0.05)$, and there was a trend toward a significant difference between the $\mathrm{SH}$ and BLA groups $(P=0.07)$. Rats with BLA lesions showed significantly greater freezing than rats with either CEA lesions or CEA + BLA lesions $(P<0.05)$, which did not differ from one another.

The group differences in conditional freezing were apparent early in training. Further analysis of the first 10 training trials (first 2 blocks; shown as an inset to Fig. 2A) with a two-way ANOVA with variables of lesions (SH, CEA, BLA, and CEA + BLA) and training trial (1-10) revealed a main effect of lesion $\left(F_{(3,47)}=23.9 ; P<0.0001\right)$ and training trial $\left(F_{(9,423)}=5.5\right.$; 


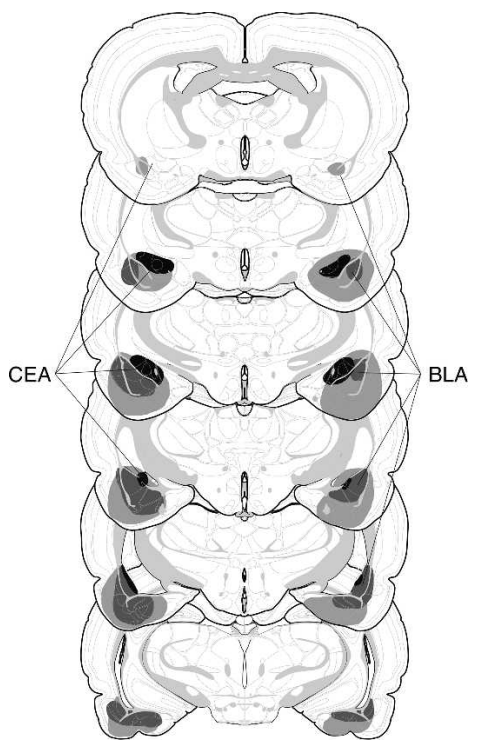

Figure 3. Schematic representation of the extent of post-training NMDA lesions (median lesion) of the CEA (black), BLA (dark gray), and CEA + BLA lesions (light gray) for Experiment 2. Coronal brain section images adapted from Swanson (1992).

$P<0.0001)$, and an interaction of lesion $\times$ training trial $\left(F_{(30,470)}=3.1 ; P<0.0001\right)$. This indicates that freezing differed among the groups during the first 10 trials of training. Post-hoc analysis of the main effect of lesion shows that sham rats exhibited significantly greater freezing than rats in any other group, and rats with BLA lesions exhibited greater freezing than rats with CEA or CEA + BLA lesions ( $P<0.05$ for all comparisons). There was no difference between rats with CEA lesions and rats with CEA + BLA lesions. As we have previously reported (Maren 1998), amygdala lesions did not affect shock reactivity to the first conditioning shock $\left(F_{(3,52)}=0.7 ; P=0.6\right)$ (data not shown). Thus, these data indicate that CEA lesions (whether alone, or in combination with BLA lesions) significantly impaired the acquisition of conditional freezing. Rats with BLA lesions exhibited more freezing than rats with CEA lesions but were also deficient relative to controls.

Long-term fear memories to the conditioning context and the auditory CS were assessed in separate retention tests conducted $24 \mathrm{~h}$ and $48 \mathrm{~h}$ after conditioning, respectively. Figure $2 \mathrm{~B}$ shows the freezing data for the context test. A two-way ANOVA with variables of lesion (SH, CEA, BLA, and CEA + BLA) and time (minutes 1-8) revealed a significant main effect of lesion $\left(F_{(3,52)}=27.8 ; P<0.0001\right)$ and time $\left(F_{(7,364)}=11.5 ; P<0.0001\right)$ without a significant interaction of lesion and time $\left(F_{(21,364)}=1.4 ; P=0.10\right)$ during the context test. Post-hoc analysis of the main effect of lesion revealed that rats with CEA lesions (alone or in combination with BLA lesions) exhibited impaired freezing compared with both BLA lesion rats and sham rats ( $P<0.05$ for all comparisons). As previously reported (Maren 1999), rats with only BLA lesions did not exhibit a significant impairment in contextual freezing when compared with sham rats, and there was no significant difference between the CEA and CEA + BLA lesion groups. These data indicate that animals with BLA lesions acquire fear to a context after overtraining and that this contextual fear is eliminated by CEA lesions.

Freezing during the tone test is shown in Figure 2C. A twoway ANOVA with variables of lesion ( $\mathrm{SH}, \mathrm{CEA}, \mathrm{BLA}$, and CEA + BLA) and time (minutes 3-8) revealed a significant main effect of lesion $\left(F_{(3,52)}=7.2 ; P<0.0004\right)$ and time $\left(F_{(5,260)}=8.8\right.$; $P<0.0001)$, and a significant interaction of lesion $\times$ time
$\left(F_{(15,260)}=3.3 ; P<0.0001\right)$. Rats with CEA lesions (alone or in combination with BLA lesions) exhibited impaired freezing compared with both BLA lesion rats and sham rats $(P<0.05)$. In contrast to an earlier report (Maren 1999), rats with BLA damage acquired freezing to the auditory CS (but see Experiment 4). Nonetheless, rats with CEA damage (either alone or in combination with BLA damage) had impaired memory to the tone CS, similar to their deficits in contextual fear.

\section{Experiment 2: Neurotoxic lesions of the CEA or BLA prevent the expression of overtrained fear}

Experiment 1 replicated earlier reports that the BLA is not an essential structure for the acquisition or expression of Pavlovian fear when animals are overtrained in an auditory fear conditioning paradigm. Indeed, the CEA appears to be critical for the acquisition of Pavlovian fear after overtraining. Because CEA lesions were made before conditioning in Experiment 1, however, it is not clear whether the effects of CEA lesions were due to impairments in the acquisition or expression of the conditional fear memory. To further explore the nature of the deficit in rats with CEA lesions, we used post-training lesions in Experiment 2 to determine whether the CEA is required for the expression of fear when the amygdala is intact during the acquisition of fear conditioning. Because recent work by Koo et al. (2004) has suggested that deficits observed in rats with CEA lesions may be the results of damage to en passant axons rather than CEA neurons, we also examined myelin staining within the amygdala to characterize the influence of NMDA on fibers of passage.

\section{Histology}

On the basis of the histological results, 14 of the 60 rats were excluded from the analysis. This yielded group sizes of CEA $(n=5)$, BLA $(n=12)$, CEA + BLA $(n=15)$, and SH $(n=14)$. The extent of the amygdala damage for rats included in the data analysis is depicted in Figure 3 and is similar to that described in Experiment 1. Figure 4 shows representative thionin- and $\mathrm{AuCl}-$
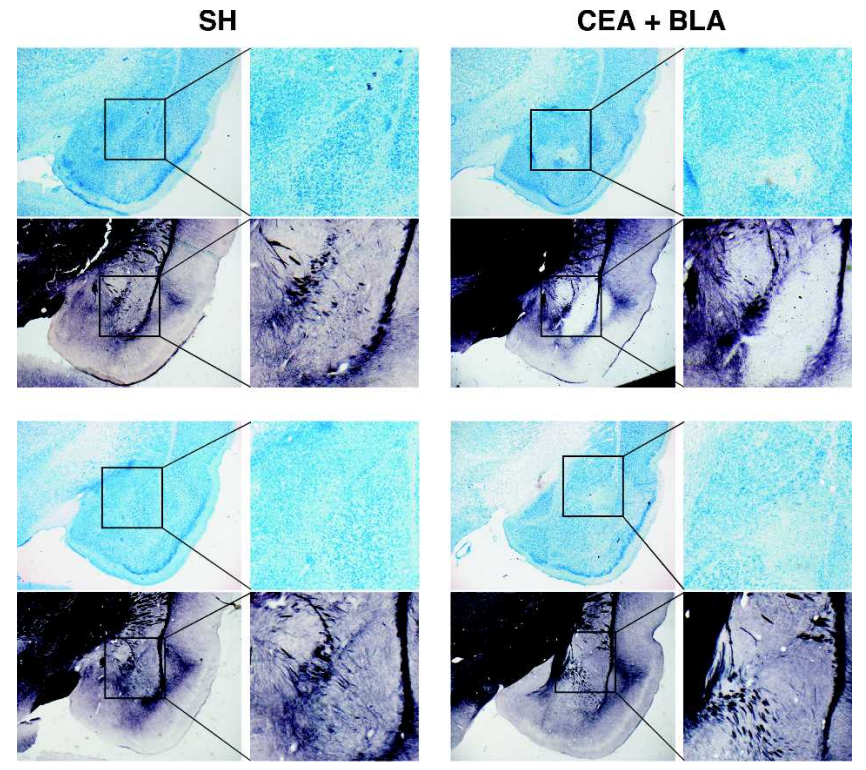

CEA

BLA

Figure 4. Representative slices stained with thionin and $\mathrm{AuCl}$. Slices shown from rats that received lesions of the CEA + BLA (upper right), CEA (lower left), or BLA (lower right); SH rats (upper left). Both the thionin- and the $\mathrm{AuCl}$-stained slices for each group are taken from the same representative animal at approximately the same anterior-posterior level with a magnification of the amygdala shown to the immediate right. 


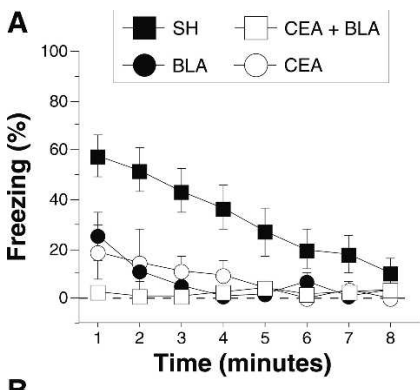

B

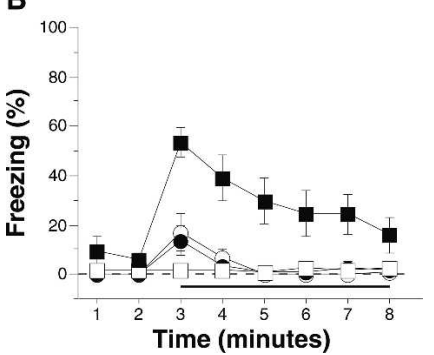

Figure 5. Conditioned freezing in rats with post-training amygdala lesions (Experiment 2). (A) Mean percentage of freezing ( \pm SEM) to contextual (8-min context extinction test) cues after at least $7 \mathrm{~d}$ of recovery from post-training surgery. (B) Mean percentage of freezing ( \pm SEM) to the auditory CS in a novel context $24 \mathrm{~h}$ after contextual testing. The auditory CS was initiated 2 min after rats were placed in the chambers (horizontal bar indicates the CS). Data are shown for rats with lesions of the BLA (solid circles), CEA (open circles), or CEA + BLA (open squares); $\mathrm{SH}$ rats (solid squares).

stained coronal sections from rats that received NMDA lesions in the CEA, BLA, or CEA + BLA, and SH rats. Relative to control tissue, myelin staining in rats with either CEA or BLA lesions appeared normal, suggesting that there was little or no damage to en passant axons. However, in rats with combined lesions of the CEA and BLA, a loss of myelin staining in the region of the lesion was observed. Therefore, the larger volumes of NMDA used to create combined lesions of the CEA and BLA yielded far more extensive damage (including fibers of passage) than lesions of either the BLA or CEA alone. Although myelin staining was apparently normal in rats with CEA lesions, it is possible that undetectable damage to the CEA in these rats contributed to their deficits. Moreover, reversible inactivation of the CEA (see Experiments 3 and 4), which presumably does not affect axonal conduction, reproduced the effects of CEA lesions.

\section{Behavior}

Post-shock freezing during the conditioning session did not differ among the groups before surgery (data not shown). Data for the context and tone retention tests, which were conducted one week after surgery, are shown in Figure 5, A and B, respectively. For the context test, a two-way ANOVA with variables of lesion (SH, CEA, BLA, and CEA + BLA) and time (minutes 1-8) revealed a significant main effect of lesion $\left(F_{(3,42)}=10.8 ; P<0.0001\right)$ and time $\left(F_{(7,294)}=4.9 ; P<0.0001\right)$. Rats with lesions of the BLA, CEA, or both CEA + BLA displayed significantly impaired freezing compared with control animals $(P<0.05)$ (Fig. 5A). Similar results were observed during the tone test (Fig. 5B). A two-way ANOVA with variables of lesion (CEA, BLA, CEA + BLA, and SH) and time (minutes 3-8) revealed a significant effect of lesion $\left(F_{(3,42)}=10.9 ; P<0.0001\right)$ and time $\left(F_{(5,210)}=12 ; P<0.0001\right)$ and lesion $\times$ time interaction $\left(F_{(15,210)}=4.3 ; P<0.0001\right)$. All groups displayed low levels of freezing before tone onset $(<10 \%)$. After tone onset animals in all of the lesion groups exhibited a significant impairment in freezing to the auditory CS compared with sham rats $(P<0.05)$. These data indicate that both the CEA and the BLA are essential for the expression of conditional freezing when animals undergo fear conditioning with both structures intact.

\section{Experiment 3: Muscimol inactivation of the CEA prevents the acquisition of overtrained fear}

Experiments 1 and 2 demonstrate that the expression of fear after overtraining is impaired in rats with lesions of the CEA, regardless of whether those lesions are made before or after training. However, pretraining lesions might influence performance by affecting either the acquisition and/or the expression of conditional freezing. Therefore, we used a temporary inactivation procedure in the following experiments to independently assess the role of the CEA in the acquisition and the expression of conditioned freezing after overtraining. Experiment 3 examined whether pretraining inactivation of the CEA with the $\mathrm{GABA}_{\mathrm{A}}$ receptor agonist muscimol impairs the acquisition of overtrained fear in rats with BLA lesions.

\section{Histology}

On the basis of the histological results, 17 of 48 rats were excluded. This yielded the following group sizes: SH-SAL $(n=7)$, SH-MUS $(n=8)$, BLA-SAL $(n=10)$, and BLA-MUS $(n=6)$. The extent of the BLA lesions as well as CEA cannula placements for rats included in the data analyses are depicted in Figure 6. All cannula placements were located in the CEA. The BLA lesions were similar to those described in Experiment 1.

\section{Behavior}

Freezing during the conditioning session, which was conducted immediately after the intra-CEA infusions, is shown in Figure 7A. The data were analyzed using a three-way ANOVA with variables of lesion (SH, BLA), drug (SAL, MUS), and trial (15 5-trial blocks). During the pretrial period rats displayed minimal levels of freezing $(<10 \%)$ before footshock. After the onset of conditioning, the

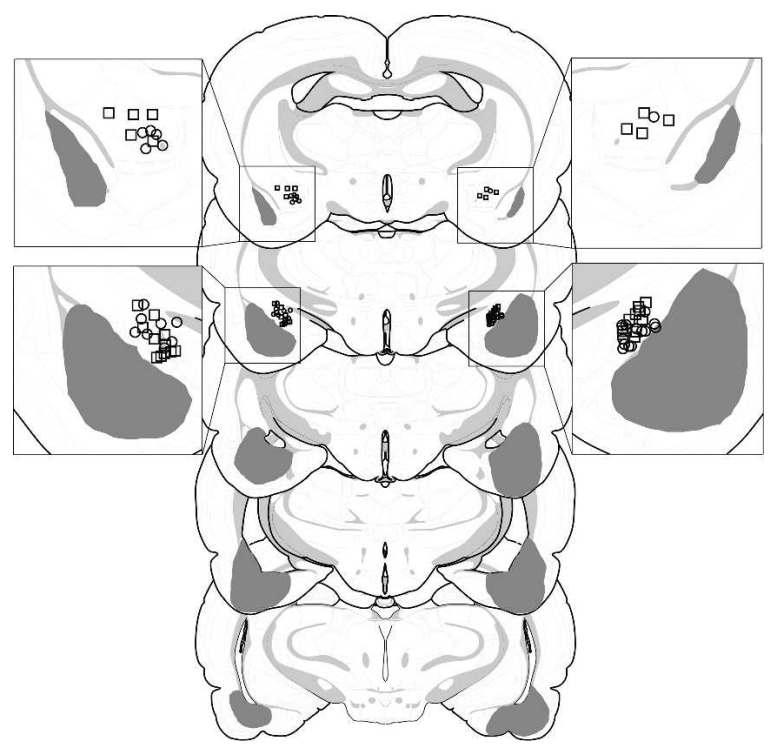

Figure 6. Schematic representation of the extent of pretraining NMDA lesions (median lesion) of the BLA (dark gray) and the locations of included cannula placements for the infusion of muscimol (circles) or $0.9 \%$ sterile saline (squares) in the CEA (Experiment 3). A magnification of the amygdala is shown in the insets adjacent to the coronal brain sections. Coronal brain section images adapted from Swanson (1992). 
A

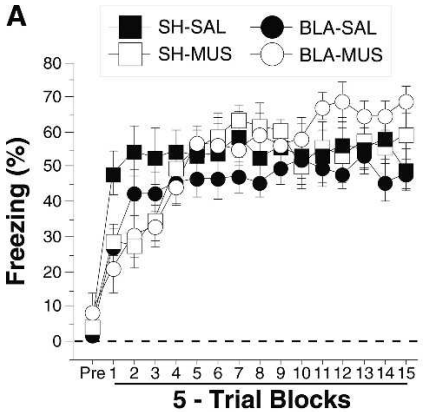

B

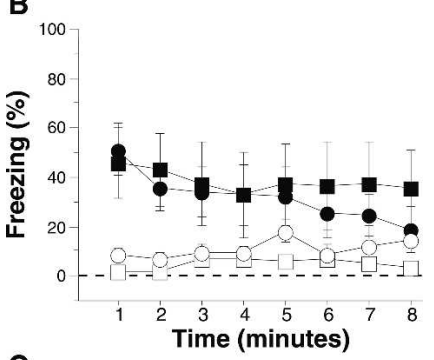

C

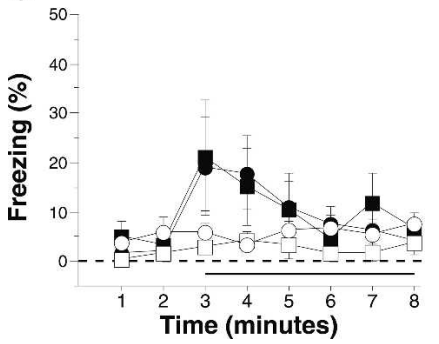

Figure 7. Conditioned freezing in rats with pretraining BLA lesions and temporary inactivation of the CEA during training (Experiment 3). (A) Mean percentage of freezing ( \pm SEM) during the 75 trial training session (data are displayed with a 3-min pretrial period followed by 15 bins consisting of 5 trials each) after infusion of $0.9 \%$ saline or muscimol into the CEA. Freezing was quantified before the first conditioning trial (Pre) and during the 1-min period after each conditioning trial; these values were averaged in 5-trial blocks. (B) Mean percentage of freezing ( \pm SEM) to contextual (8-min context extinction test) cues $24 \mathrm{~h}$ after training. (C) Mean percentage of freezing ( \pm SEM) to the auditory CS in a novel context $48 \mathrm{~h}$ after training. The auditory CS was initiated 2 min after rats were placed in the chambers (horizontal bar indicates the CS). Data are shown for rats with pretraining sham surgeries receiving saline in the CEA before training (SH-SAL: solid squares), pretraining sham surgeries receiving muscimol in the CEA before training (SH-MUS: open squares), pretraining NMDA lesions of the BLA receiving saline in the CEA before training (BLA-SAL: solid circles), or pretraining NMDA lesions of the BLA receiving muscimol in the CEA before training (BLA-MUS: open circles).

rats exhibited robust freezing. There was neither a main effect of lesion $\left(F_{(1,27)}=0.3 ; P=0.6\right)$ or drug $\left(F_{(1,27)}=0.2 ; P=0.6\right)$, nor a lesion $\times$ drug interaction $\left(F_{(1,27)}=1.0 ; P=0.3\right)$ on conditional freezing after the onset of conditioning. However, the ANOVA revealed a significant main effect of trial $\left(F_{(14,378)}=17.5\right.$; $P<0.0001)$, a significant lesion $\times$ trial interaction $\left(F_{(14,378)}=7.1 ; P=0.01\right)$, and a significant drug $\times$ trial interaction $\left(F_{(14,378)}=7.1 ; P<0.0001\right)$. This indicates that although the overall levels of freezing were unaffected by either the lesion or drug, the rates of acquisition were affected by both lesion and drug treatment. Furthermore, as shown in Figure 8, muscimol treatment did not affect the activity burst elicited by the first conditioning shock as measured by ANOVA with variables of lesion (SH, BLA), drug (SAL, MUS), and shock reactivity (pretrial activity, shock activity). This analysis revealed only a significant main effect of shock reactivity $\left(F_{(1,27)}=1.0 ; P<0.0001\right)$, indicat- ing an equivalent burst of activity for each group during the first footshock.

Data from the context and tone retention tests, which were conducted drug-free 24 and $48 \mathrm{~h}$ after conditioning, are shown in Figure 7, B and C, respectively. For the context test, an ANOVA with variables of lesion (SH, BLA), drug (SAL, MUS), and time (minutes 1-8) revealed a significant main effect of drug $\left(F_{(1,27)}=9.3 ; P<0.01\right)$ and a significant drug $\times$ time interaction $\left(F_{(7,189)}=2.2 ; P<0.04\right)$ without significant main effects of lesion $\left(F_{(1,27)}<0.01 ; P=1.0\right)$ or time $\left(F_{(7,189)}=1.0 ; P=0.4\right)$. This indicates that rats for which the CEA was inactivated during training (SH-MUS, BLA-MUS) showed significantly less freezing than rats that received saline infusions into the CEA during training $(\mathrm{SH}-$ SAL, BLA-SAL) regardless of whether the BLA was damaged (Fig. 7B). A similar effect was observed during the tone test (Fig. 7C). All groups displayed low levels of freezing before tone onset $(<5 \%)$, and, in this particular experiment, freezing to the tone CS was also unusually low. For this reason, we focused the analysis on the first two minutes of the tone test, a period during which freezing to the tone CS was greater than baseline. An ANOVA with variables of lesion (SH, BLA) and drug (SAL, MUS) revealed a significant main effect of drug $\left(F_{(1,27)}=4.1 ; P=0.05\right)$ without a significant main effect of lesion $\left(F_{(1,27)}<0.01 ; P=0.93\right)$ or a lesion $\times$ drug interaction $\left(F_{(1,27)}<0.01 ; P=0.97\right)$. Thus, during the first two minutes of the tone CS, rats infused with muscimol during training (SH-MUS, BLA-MUS) showed significantly less freezing that saline-infused rats (SH-SAL, BLA-SAL). Although freezing to the tone CS was lower than normal in the salinetreated rats, it is clear that the muscimol infusion into the CEA impeded acquisition. These data indicate that the CEA is critical for the acquisition of conditioned fear in rats with BLA lesions and that CEA inactivation in intact rats also impairs the acquisition of fear conditioning.

\section{Experiment 4: Muscimol inactivation of the CEA prevents the expression of overtrained fear}

Experiment 3 demonstrates the necessity of the CEA for the acquisition of conditioned fear following overtraining. Experiment 4 examined whether CEA inactivation also impairs the expression of conditioned freezing in rats overtrained after receiving BLA lesions.

\section{Histology}

On the basis of the histological results, 11 of the 40 rats were excluded. Exclusions were made because the lesions were smaller

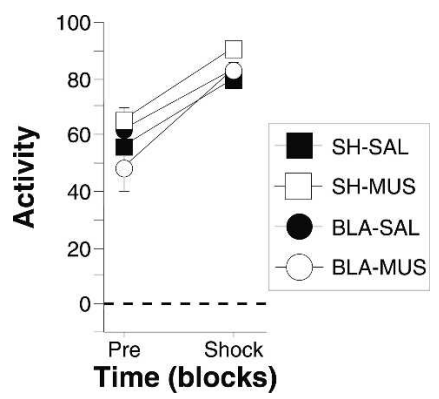

Figure 8. Shock reactivity in rats with pretraining BLA lesions and temporary inactivation of the CEA before training (Experiment 3). Mean percentage of activity ( \pm SEM) before the first conditioning trial (Pre) and during the first 2-sec shock (Shock) during the conditioning session. Data are shown for rats with pretraining sham surgeries receiving saline in the CEA before training (SH-SAL: solid squares), pretraining sham surgeries receiving muscimol in the CEA before training (SH-MUS: open squares), pretraining NMDA lesions of the BLA receiving saline in the CEA before training (BLA-SAL: solid circles), or pretraining NMDA lesions of the BLA receiving muscimol in the CEA before training (BLA-MUS: open circles). 
or larger than intended or were misplaced or if the cannula was improperly placed in the CEA. One animal that displayed a marked motor deficit after muscimol infusion was also excluded. This yielded samples sizes per group of SH-SAL $(n=12)$, SH-MUS $(n=6)$, BLA-SAL $(n=5)$, and BLA-MUS $(n=6)$. The extent of the BLA lesions as well as CEA cannula placements for rats included in the data analyses are depicted in Figure 9. All cannula placements were located in the CEA. BLA lesions were similar to those described in Experiment 1.

\section{Behavior}

Post-shock freezing during the conditioning session is shown in Figure 10A. Similar to Experiment 1, post-shock freezing was significantly lower in rats with BLA lesions as compared with sham controls. Two-way ANOVA with variables of lesion (SH, BLA) and training trial block (15 5-trial blocks) confirmed this via a main effect of lesion $\left(F_{(1,27)}=10.4 ; P<0.01\right)$ and training block $\left(F_{(14,378)}=3.2 ; P<0.0001\right)$.

Conditional freezing during the context and tone retention tests is shown in Figure 10, B and C, respectively. Animals receiving muscimol infusions into the CEA showed a marked reduction in conditional freezing to the context. An ANOVA with variables of lesion (SH, BLA), drug (SAL, MUS), and time (minutes 1-8) revealed a main effect of drug $\left(F_{(1,25)}=13.1 ; P<0.01\right)$, indicating that rats receiving muscimol in the CEA exhibited significantly lower freezing in both intact rats and rats with BLA lesions. Importantly, neither a significant main effect of lesion $\left(F_{(1,25)}<0.02 ; P=0.89\right)$ nor a significant lesion $\times$ drug interaction $\left(F_{(1,25)}<0.03 ; P>0.88\right)$ was observed, indicating that BLA lesions did not prevent the acquisition of context fear after overtraining (Experiment 1; Maren 1999).

During the tone test, freezing was greatly reduced in animals receiving muscimol infusions into the CEA. An ANOVA with variables of lesion (SH and BLA), drug (SAL and MUS), and time (minutes 3-8) revealed a significant drug $\times$ time interaction $\left(F_{(5120)}=2.9 ; P<0.02\right)$; no other effects reached significance. Post-hoc comparisons revealed higher levels of freezing in salineinfused rats compared with muscimol-infused groups in the first

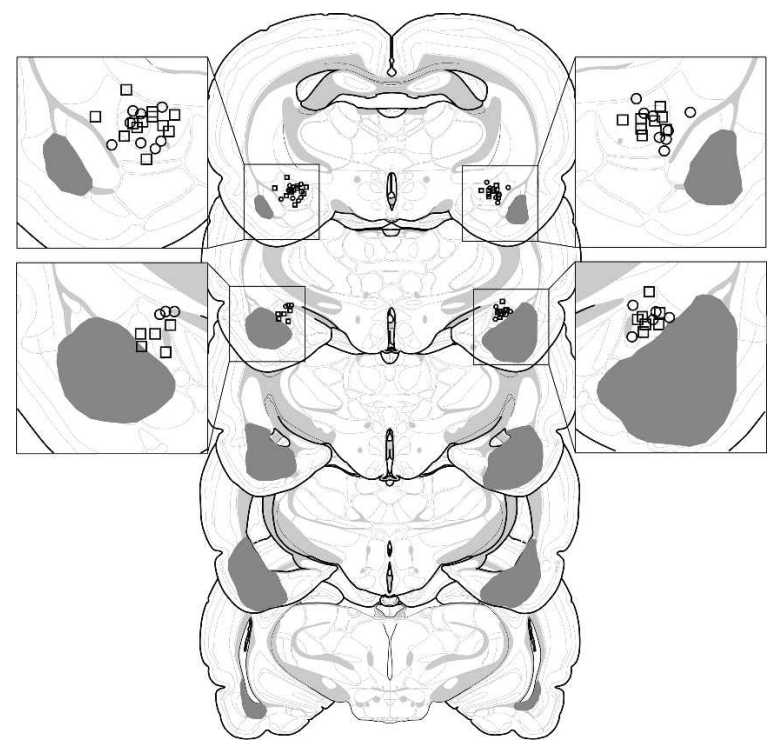

Figure 9. Schematic representation of the extent of pretraining NMDA lesions (median lesion) of the BLA (dark gray) and the locations of included cannula placements for the infusion of muscimol (circles) or $0.9 \%$ sterile saline (squares) in the CEA (Experiment 4). A magnification of the amygdala is shown adjacent to the coronal brain sections. Coronal brain section images adapted from Swanson (1992).
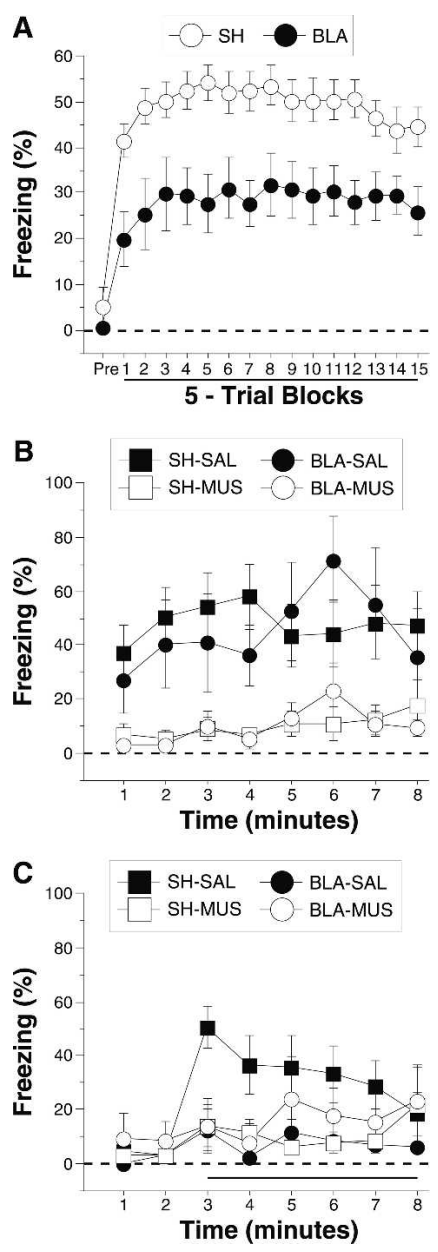

Figure 10. Conditioned freezing in rats with pretraining BLA lesions and temporary inactivation of the CEA during testing (Experiment 4). (A) Mean percentage of freezing ( \pm SEM) during the 75 trial training session (data are displayed with a 3-min pretrial period followed by 15 bins consisting of 5 trials each). Freezing was quantified before the first conditioning trial (Pre) and during the 1-min period after each conditioning trial; these values were averaged in 5 -trial blocks. $(B)$ Mean percentage of freezing $( \pm$ SEM) to contextual (8-min context extinction test) cues after infusion of $0.9 \%$ sterile saline or muscimol into the CEA. (C) Mean percentage of freezing ( \pm SEM) to the auditory CS in a novel context $48 \mathrm{~h}$ after training after infusion of $0.9 \%$ sterile saline or muscimol into the CEA. The auditory CS was initiated 2 min after rats were placed in the chambers (horizontal bar indicates the CS). Data are shown for rats with pretraining sham surgeries receiving saline in the CEA before testing ( $\mathrm{SH}$ SAL: solid squares), pretraining sham surgeries receiving muscimol in the CEA before testing (SH-MUS: open squares), pretraining NMDA lesions of the BLA receiving saline in the CEA before testing (BLA-SAL: solid circles), or pretraining NMDA lesions of the BLA receiving muscimol in the CEA before testing (BLA-MUS: open circles).

two minutes of the test. This result indicates that rats receiving muscimol infusions into the CEA exhibited impairments in the expression of conditional freezing to the auditory CS as compared with the SH-SAL group. Although there was not a statistically significant effect of the lesion in the overall ANOVA, it is apparent that rats with BLA lesions exhibited considerably less freezing than saline-infused SH controls. To assess this we separately analyzed freezing during the first two-minutes of the test in the saline-infused groups. During this period, BLA rats infused with saline exhibited significantly less freezing than $\mathrm{SH}$ rats $\left(F_{(1,15)}=6.2 ; P<0.05\right)$. The failure of BLA rats to acquire auditory freezing after overtraining has been observed in earlier reports 
(Maren 1999). Together, these data indicate that pharmacological inactivation of the CEA blocks the expression of overtrained fear to either contextual or auditory stimuli in both intact rats and rats with BLA lesions.

\section{Discussion}

The present experiments used an overtraining procedure to determine the role of the CEA in the acquisition and expression of Pavlovian fear conditioning in rats with neurotoxic BLA lesions. The main outcome of the experiments was that permanent or temporary inactivation of the CEA in rats with BLA lesions prevented both the acquisition and expression of conditioned freezing to the conditioning context and an auditory CS after a 75trial overtraining procedure. Thus, the ability of rats with BLA lesions to acquire conditioned freezing (e.g., Maren 1999) appears to depend on the integrity of the CEA. Moreover, similar to the findings of Wilensky et al. (2006), the CEA appears to be involved in the acquisition of conditioned fear in rats with an intact BLA, insofar as reversible inactivation of the CEA impaired the acquisition of conditioned freezing in both intact rats and rats with BLA lesions. Both the BLA and CEA appear to be essential for the expression of conditioned freezing acquired after overtraining, because post-training lesions of either structure eliminated conditioned freezing.

These data replicate and extend earlier observations that extensive overtraining promotes conditioning in rats with neurotoxic BLA lesions (Maren 1999; Lee et al. 2005). Less extensive training (e.g., 25 trials) produces a modest level of fear conditioning in rats with BLA lesions (Maren 1998; Lee et al. 2005), but it is now clear that considerably more training is required to obtain the high levels of conditioned freezing that are typically observed in intact animals after only a few trials. Similar to the findings of Cahill et al. (2000), rats with BLA lesions acquired conditioned freezing at a significantly lower rate than intact rats during the conditioning session. Despite a reduction in postshock freezing, rats with BLA lesions exhibited robust conditioned freezing to the conditioning context (and in one experiment the auditory CS) during retention testing. In contrast, rats with CEA lesions, either alone or in combination with BLA lesions, expressed low levels of conditional freezing during both conditioning and retention testing despite overtraining.

Kim and Davis (1993) have also found that rats with CEA lesions are unable to acquire fear-potentiated startle even after extensive training. Because we found that reversible inactivation of the CEA prevented the acquisition and expression of conditional freezing in both intact rats and rats with BLA lesions, these results suggest that the CEA is necessary for both the acquisition and expression of conditional freezing, even after extensive overtraining. Interestingly, rats with CEA lesions can reacquire a fear-potentiated startle response if they are extensively trained before the lesion. This suggests that the BLA, which continues to be important for the expression of conditioned fear even after overtraining (Falls and Davis 1995; Maren 1998, 1999), might be able to control the performance of fear CRs in the absence of the CEA.

The critical role for the CEA in the expression of conditional fear has been recognized in numerous studies (Campeau and Davis 1995; Goosens and Maren 2001). Our results, indicating that discrete lesions of the CEA block the expression of conditioned fear, extend this important role of the CEA in fear conditioning to overtrained fear. However, our results are at odds with a recent report that found that rats with fiber-sparing ibotenic acid lesions of the CEA exhibited normal contextual freezing and only slightly attenuated fear to an auditory CS (despite showing a deficit in conditioned ultrasonic vocalizations) after 10-trial fear conditioning (Koo et al. 2004). The reason for this discrepancy is not clear, insofar as we obtained reliable deficits in conditioned freezing with both fiber-sparing CEA lesions (see Experiments 1and 2) and muscimol infusions into the CEA (see Experiments 3 and 4), and have previously observed deficits in conditioned freezing with small ibotenic acid lesions in the CEA (Goosens and Maren 2001). It is possible that the precise locus or extent of the lesions within the CEA differ between these studies. Indeed, the lesions made by Koo et al. (2004) were made with iontophoretic methods that may have yielded smaller lesions than the pressure injections of NMDA used in the present study. Nevertheless, we also obtained deficits in conditional freezing after muscimol infusions into the CEA, suggesting that neurons in the CEA are not only critical for the expression, but also the acquisition, of conditional fear. This finding is further supported by the work of Wilensky et al. (2006) using a 2-trial fear conditioning procedure. The fact that our deficits were obtained in rats with BLA lesions argues against the possibility that infusions of muscimol into the CEA produced the effects by diffusing to the neighboring BLA.

Despite exhibiting deficits in the expression of conditional freezing during retention testing, rats with CEA inactivation or lesions exhibited substantial levels of conditional freezing during the conditioning session (see Experiment 1 and Experiment 3). It has been argued that freezing behavior shortly after footshock is a conditioned response to the conditioning context, rather than an unconditioned response to footshock (Fanselow 1990). If so, our data suggest that short-term conditioned fear responses may survive, at least in part, CEA lesions or inactivation. Alternatively, freezing during the conditioning session may represent an unconditioned response to footshock (Bevins et al. 1997). In either case, it may be that other brain areas that are essential for freezing behavior, such as the periaqueductal gray, are involved in the expression of fear responses (whether conditioned or unconditioned) shortly after footshock. However, our data suggest that the CEA is ultimately critical for the expression of conditioned freezing driven by the long-term memory of the conditioning experience.

Interestingly, the ability of the CEA to compensate for loss of the BLA appears to be engaged by overtraining. That is, rats with neurotoxic BLA lesions exhibit substantial deficits in conditioned freezing with either 1 or 25 conditioning trials (Maren $1998,1999)$. It is only when these rats are given extensive overtraining (50 or 75 conditioning trials) that they exhibit conditioned freezing that is similar in magnitude to that in control rats. It is important to note that the CEA only compensates for the loss of the BLA if conditioned fear is acquired in the absence of the BLA (Experiment 2), a finding similar to that of AngladaFigueroa and Quirk (2005). Two possibilities might account for this pattern of results. First, the CEA might only be involved in the expression of fear driven by memory acquired in other brain areas. If so, then these other areas are either inefficient in acquiring fear memory in the absence of the BLA or insufficient to drive the performance of fear responses via the CEA. However, Experiment 3 suggests that the CEA itself may be involved in the encoding of fear memory, because CEA inactivation in intact rats impaired the acquisition of conditional freezing, a finding consistent with the recent work of Wilensky et al. (2006). If the CEA is the primary site of memory encoding in the absence of the BLA, it would appear to be less efficient than the BLA in coding fear memories insofar as it required substantially more training to elicit conditioned freezing in rats with BLA lesion. It might also be the case that the associative representations mediated by the CEA and BLA are different (Killcross et al. 1997; Holland and Gallagher 2003; Balleine and Killcross 2006) and that those mediated by the CEA can come to generate conditioned freezing, 
but only after extensive training. Studies are underway to explore this possibility.

Our data suggest a role for the CEA not only in the expression but also in the acquisition of long-term fear memory (or at least the fear behavior engendered by such memories), a role formerly limited to the BLA (Pare et al. 2004; Maren 2005b). Intact animals receiving muscimol inactivation of the CEA during conditioning showed significant deficits in conditioned fear to both contextual and auditory cues $24 \mathrm{~h}$ and $48 \mathrm{~h}$, respectively, after conditioning (Experiment 3). Additionally, we have recently reported that infusions of an NMDA receptor antagonist into the CEA prevents the acquisition of conditional freezing (5 tone-footshock pairings), although these rats did exhibit some savings when they received additional training in a drug-free state (Goosens and Maren 2003). Although we have not observed impairments in conditioned freezing after the infusion of a broad-spectrum protein kinase inhibitor (Goosens et al. 2000) or a Ras antagonist (Merino and Maren 2006) into the CEA, others have reported deficits after infusions of either a protein kinase A inhibitor or a protein synthesis inhibitor into the CEA (Wilensky et al. 2006). These data suggest that cellular mechanisms involved in long-term memory and synaptic plasticity might operate in the CEA to encode fear memory. Further work is necessary to explore this possibility.

In summary, we have found that the CEA is essential for the acquisition and expression of conditioned freezing after overtraining. Moreover, our results reveal that the capacity of rats with neurotoxic BLA lesions to acquire fear after overtraining is mediated by the CEA. These results therefore provide additional support for the parallel processing model within the amygdala through which associative functions are mediated by both the BLA and CEA, as animals are able to learn in the absence of the BLA (a structure essential for learning according the to serial processing model). Furthermore, our data suggest an unequal weighting in the parallel pathways, as rats trained in the absence of the BLA require substantially more training (extensive overtraining) to acquire substantial conditioned fear. Moreover, rats trained with an intact BLA lose their fear memory after the BLA is lesioned, even after extensive overtraining. It is not clear whether the nature of the associations encoded by the CEA is similar to that of the BLA. Nonetheless, these data reveal an important role for the CEA in mediating overtrained fear and support the emerging view that associative processes in the CEA might contribute to fear conditioning under some conditions.

\section{Materials and Methods}

\section{Experiment 1: Neurotoxic CEA lesions prevent the acquisition of overtrained fear}

\section{Subjects}

The subjects were 74 male Long-Evans rats (200-224 g; Blue Spruce) obtained from a commercial supplier (Harlan Sprague Dawley). After arrival, the animals were individually housed in clear plastic cages hanging from a standard stainless-steel rack. The vivarium lights were on a 14/10 light/dark cycle (lights on at 7:00 am) and the rats had free access to food and tap water. After housing, the rats were handled (15-20 sec each) for five days to acclimate them to the experimenter. All experiments were carried out in accordance with guidelines approved by the University of Michigan University Committee on Use and Care of Animals.

\section{Behavioral apparatus}

Eight identical observation chambers $(30 \times 24 \times 21 \mathrm{~cm}$; MedAssociates) were used for all phases of training and testing. The chambers were constructed from aluminum (two side walls) and
Plexiglas (rear wall, ceiling, and hinged front door) and were situated in sound-attenuating chests located in an isolated room. The floor of each chamber consisted of 19 stainless-steel rods (4 $\mathrm{mm}$ diameter) spaced $1.5 \mathrm{~cm}$ apart (center to center). The rods were wired to a shock source and solid-state grid scrambler (MedAssociates) for delivery of the footshock (US) $(1.0 \mathrm{~mA}, 2 \mathrm{sec})$. For "context A" (used for conditioning and context retention testing), background noise ( $65 \mathrm{~dB}$ ) was provided by ventilation fans built into the chests, house lights within the chambers and fluorescent lights within the room provided illumination, the chest doors were left open, and the chambers were cleaned with a $1 \%$ ammonium hydroxide solution. For "context B" (used for tone retention testing), illumination was provided by fluorescent red lights, the chest doors were closed, the ventilation fans were inactive, and the chambers were cleaned with a $1 \%$ acetic acid solution. Stainless steel pans containing a thin film of the corresponding cleaning solutions were placed underneath the grid floors before the animals were placed inside the boxes.

Each conditioning chamber rested on a load cell platform that was used to record chamber displacement in response to each rat's motor activity. To ensure interchamber reliability, each load cell amplifier was calibrated to a fixed chamber displacement. The output of the load cell of each chamber was set to a gain that was optimized for detecting freezing behavior. Load cell amplifier output from each chamber was digitized and acquired on-line using Threshold Activity software (Med-Associates).

\section{Surgery}

The rats were randomly assigned to groups that received bilateral neurotoxic lesions of the BLA, CEA, or combined lesions of both the BLA and CEA (BLA + CEA); control rats received sham (SH) surgery. After handling for at least $5 \mathrm{~d}$, rats were treated with atropine sulfate $(0.4 \mathrm{mg} / \mathrm{kg}$ body weight, i.p. $)$ and sodium pentobarbital (Nembutal, $65 \mathrm{mg} / \mathrm{kg}$ body weight, i.p.) and mounted in stereotaxic apparatus (David Kopf instruments). The scalp was incised and retracted, and the head was positioned to place bregma and lambda in the same horizontal plane. Small burr holes ( $2 \mathrm{~mm}$ in diameter) were drilled bilaterally in the skull for the placement of 28-gauge cannula in the BLA $(3.3 \mathrm{~mm}$ posterior to bregma, $5.0 \mathrm{~mm}$ lateral to the midline), CEA (2.3 $\mathrm{mm}$ and 2.7 $\mathrm{mm}$ posterior to bregma, $4.3 \mathrm{~mm}$ lateral to the midline), or both. Two 10- $\mu \mathrm{L}$ Hamilton syringes were mounted into an infusion pump (Harvard Apparatus) and connected to the injection cannula with polyethylene tubing. NMDA was dissolved in $100 \mathrm{mM}$ PBS (20 mg/mL at pH 7.4; Sigma). For BLA lesions, NMDA was infused $(0.1 \mu \mathrm{L} / \mathrm{min})$ at two sites: $8.0 \mathrm{~mm}$ ventral to brain surface $(0.2 \mu \mathrm{L})$ and $7.5 \mathrm{~mm}$ ventral to brain surface $(0.1 \mu \mathrm{L})$. For CEA lesions, NMDA was infused $(0.1 \mu \mathrm{L} / \mathrm{min}) 7.9 \mathrm{~mm}$ ventral to brain surface $(0.15 \mu \mathrm{L})$ at each of the anterior-posterior coordinates. Five minutes were allowed after each infusion for diffusion of the drug. Sham animals received a similar surgery and had small burr holes drilled bilaterally in their skulls, but injectors were not lowered into the brain. After surgery, the incision was closed with stainless steel wound clips, and the rats were allowed to recover on a heating pad before returning to their home cage.

\section{Procedure}

After surgery, the rats were allowed at least $7 \mathrm{~d}$ to recover. On the conditioning day, the rats were transported to the laboratory in squads of eight and placed in the conditioning chambers. The chamber position was counterbalanced for each squad and group. The rats received 75 tone $(80 \mathrm{~dB}, 10 \mathrm{sec}, 2 \mathrm{kHz})$-shock $(1.0$ $\mathrm{mA}, 2.0 \mathrm{sec}$ ) pairings (70-sec intertrial interval) beginning $30 \mathrm{sec}$ after being placed in the chamber (this interval was $3 \mathrm{~min}$ for Experiments 2-4) and ending $60 \mathrm{sec}$ after the final shock (context A). Twenty-four hours after training, contextual fear was assessed by returning the rats to the conditioning chambers and measuring freezing behavior (somatomotor immobility except that necessitated by breathing) during an 8-min extinction test (context A). Forty-eight hours after training, conditional fear to the tone CS was assessed by placing the rats in a novel context (context B) and measuring freezing behavior during an extinction test in 
which a 6 -min continuous tone was presented 2 min after the rats were placed in the chambers.

During both the conditioning and test sessions, each rat's activity was monitored continuously using the data acquisition software described above. For each chamber, load cell activity was digitized at $5 \mathrm{~Hz}$, yielding one observation per rat every 200 msec (300 observations per rat per minute). Load cell values ranged between 0 and 100, and this value was used to quantify locomotor activity. Freezing was quantified by computing the number of observations for each rat that had a load cell value less than the freezing threshold (threshold $=10$ ). The freezing threshold was determined in a separate group of pilot animals by comparing load cell output with an observer's rating of freezing behavior. To avoid counting momentary inactivity as freezing, an observation was only scored as freezing if it fell within a contiguous group of at least five observations that were all less than the freezing threshold. Thus, freezing was only scored if the rat was immobile for at least $1 \mathrm{sec}$. For each session, the freezing observations were transformed to a percentage of total observations. In the present experiment, freezing was quantified before footshock during the pretrial period and after footshock offset on the conditioning day, and during the 8-min context and tone extinction tests.

\section{Histology}

Histological verification of lesion location was performed after behavioral testing. Rats were perfused across the heart with $0.9 \%$ saline followed by $10 \%$ formalin. After extraction from the skull, the brains were post-fixed in 10\% formalin for $2 \mathrm{~d}$ and $10 \%$ formalin and $30 \%$ sucrose until sectioning. Coronal sections (45 $\mu \mathrm{m}$ thick, taken every $135 \mu \mathrm{m})$ were cut on a cryostat $\left(-20^{\circ} \mathrm{C}\right)$ and wet mounted on glass microscope slides with 70\% ethanol. After drying, the sections were stained with $0.25 \%$ thionin to visualize neuronal cell bodies. Lesions were verified by visual inspection of the stained brain sections.

\section{Data analysis}

For each session, the freezing data were transformed to a percentage of total observations, a probability estimate that is amenable to analysis with parametric statistics. These probability estimates of freezing were analyzed using ANOVA. Post-hoc comparisons in the form of Fisher's PLSD tests were performed after a significant overall $F$ ratio. All data are represented as mean \pm SEM.

\section{Experiment 2: Neurotoxic lesions of the CEA or BLA prevent the expression of overtrained fear}

\section{Subjects}

The subjects were 60 adult male Long-Evans rats (200-224 g; Blue Spruce) obtained and housed as described in Experiment 1.

\section{Apparatus, surgery, and procedure}

The behavioral apparatus and conditioning procedures were identical to those described in Experiment 1, except that rats received lesions 1-4 d after conditioning (rather than 1 wk before conditioning as in Experiment 1). The rats were randomly assigned to groups that were to receive post-training lesions of the BLA, CEA, or combined lesions of both BLA and CEA (BLA + CEA); control rats received sham (SH) surgery. After surgery, the animals were allowed at least $7 \mathrm{~d}$ for recovery. Fear conditioning to the conditioning context and auditory CS was assessed as described in Experiment 1 . The rats' activity and freezing were measured and quantified as described in Experiment 1.

\section{Histology}

Rats were perfused and the intact brain was prepared as described in Experiment 1. Alternate coronal sections $(45 \mu \mathrm{m}$ thick slices, taken every $135 \mu \mathrm{m})$ were cut on a cryostat $\left(-20^{\circ} \mathrm{C}\right)$. The first slice was wet mounted on glass microscope slides with $70 \%$ ethanol to be stained with $0.25 \%$ thionin for histological verification of lesions. The second slice was stored at $4^{\circ} \mathrm{C}$ in a cryoprotective buffer containing $25 \%$ ethylene glycol, $25 \%$ glycerin, and $0.05 \mathrm{M}$ phosphate buffer. Myelin staining was preformed as described by Koo et al. (2004). Sections were washed free-floating $3 \times 10 \mathrm{~min}$ in $0.02 \mathrm{M}$ PBS $(0.6 \% \mathrm{NaCl})$. Slices were then incubated in a $0.2 \%$ $\mathrm{AuCl}$ solution containing gold chloride trihydrate, $30 \% \mathrm{H}_{2} \mathrm{O}_{2}$, and $0.02 \mathrm{M}$ PBS $(0.6 \% \mathrm{NaCl})$ until the fibers in the amygdala contrasted with the background tissue $(\sim 2 \mathrm{~h})$. Rinsing the tissue for $10 \mathrm{~min}$ in normal saline stopped the reaction. After the saline rinse, the tissue was fixed for $5 \mathrm{~min}$ in a $5 \%$ sodium thiosulfate solution. Tissue was next rinsed $3 \times 5 \mathrm{~min}$ in $0.02 \mathrm{M}$ PBS $(0.6 \%$ $\mathrm{NaCl})$ and mounted on unsubbed glass microscope slides and dried overnight at $37^{\circ} \mathrm{C}$. On the following day, tissue was dehydrated and covered with a coverslip. Fiber staining was assessed by visual inspection of the sections under a light microscope.

\section{Data analysis}

Data analysis was performed as described in Experiment 1.

\section{Experiment 3: Muscimol inactivation of the CEA prevents the acquisition of overtrained fear}

\section{Subjects}

The subjects were 48 adult male Long-Evans rats (200-224 g) obtained and housed as described in Experiment 1.

\section{Surgery}

After handling for at least $5 \mathrm{~d}$, rats were treated with atropine sulfate $(0.4 \mathrm{mg} / \mathrm{kg}$ body weight, i.p. $)$ and sodium pentobarbital (Nembutal, $65 \mathrm{mg} / \mathrm{kg}$ body weight, i.p.) and mounted in stereotaxic apparatus (David Kopf instruments). The scalp was incised and retracted, and head position was adjusted to place bregma and lambda in the same horizontal plane. Small burr holes (2 $\mathrm{mm}$ in diameter) were drilled bilaterally in the skull for the placement of 28-gauge cannula in the BLA $(3.3 \mathrm{~mm}$ posterior to bregma, $5.0 \mathrm{~mm}$ lateral to the midline) for rats receiving BLA lesions. In addition, all rats were implanted with Plastics One 26-gauge guide cannula (cut at $11 \mathrm{~mm}$ below the pedestal) into the CEA $(2.5 \mathrm{~mm}$ posterior to bregma, $4.3 \mathrm{~mm}$ lateral to the midline). BLA lesions were made as described in Experiment 1. Sham animals did not receive BLA lesions but were implanted with guide cannulas in the CEA as described above. After implantation, dental acrylic was applied to the skull to hold the cannulas in place. After surgery, dummy cannulas (33-gauge, $16 \mathrm{~mm}$; Plastics One) were inserted into the guide cannulas, and the rats were returned to their home cages. The dummy cannulas were replaced every other day during the week of recovery.

\section{Apparatus and procedure}

The behavioral apparatus and training procedures were identical to those described in Experiment 1, except that infusions of either saline or muscimol were made into the CEA before conditioning. All retention testing was performed drug-free as described in Experiment 1 with context and tone tests $24 \mathrm{~h}$ and 48 $\mathrm{h}$ after conditioning, respectively. The rats were randomly assigned to groups in a $2 \times 2$ design (lesion $\times$ drug). This design yielded the following groups: rats with pretraining sham surgeries receiving saline in the CEA (SH-SAL), rats with pretraining sham surgeries receiving muscimol in the CEA (SH-MUS), rats with pretraining NMDA lesions of the BLA receiving saline in the CEA (BLA-SAL), and rats with pretraining NMDA lesions of the BLA receiving muscimol in the CEA (BLA-MUS).

After at least $7 \mathrm{~d}$ recovery from surgery, rats were acclimated to the infusion procedure by transporting them to the infusion room in identical white 5-gallon buckets in squads of eight (counterbalanced for each squad and group). Their dummy cannulas were replaced and the infusion pumps (Harvard Apparatus) were activated. After $2.5 \mathrm{~min}$, the pumps were stopped and the animals were returned to their home cages. Twenty-four hours after acclimation, the rats were transported to the infusion room as described above and infused with either muscimol $(0.125 \mu \mathrm{g}$ in $0.25 \mu \mathrm{L}$ of sterile saline at $0.1 \mu \mathrm{L} / \mathrm{min})$ or sterile saline $(0.9 \% ; 0.25$ $\mu \mathrm{l}$ at $0.1 \mu \mathrm{L} / \mathrm{min}$ ). After the infusion, $1 \mathrm{~min}$ was allowed for diffusion before the internal cannulas were removed. After the 
internal cannulas were removed, clean dummy cannulas were inserted into the guide cannulas, and rats were immediately transported to the conditioning chambers as described in Experiment 1 , where they received auditory fear conditioning. Fear to the conditioning context and auditory CS were assessed 24 and $48 \mathrm{~h}$ later respectively, as described in Experiment 1.

\section{Histology}

Histological verification of lesions and cannula placement location was performed after behavioral testing and completed as described in Experiment 1.

\section{Data Analysis}

Data analysis was performed as described in Experiment 1.

\section{Experiment 4: Muscimol inactivation of the CEA prevents the expression of overtrained fear}

\section{Subjects}

The subjects were 48 adult male Long-Evans rats (200-224 g; Blue Spruce) obtained and housed as described in Experiment 1.

\section{Surgery}

Surgeries were performed as described in Experiment 3.

\section{Apparatus and procedure}

The behavioral apparatus and contexts were identical to that described in Experiment 1 . The rats were randomly assigned to groups in a $2 \times 2$ design (lesion $\times$ drug). This design yielded the following groups: rats with pretraining sham surgeries receiving saline in the CEA before testing (SH-SAL), rats with pretraining sham surgeries receiving muscimol in the CEA before testing (SH-MUS), rats with pretraining NMDA lesions of the BLA receiving saline in the CEA before testing (BLA-SAL), and rats with pretraining NMDA lesions of the BLA receiving muscimol in the CEA before testing (BLA-MUS). After $5 \mathrm{~d}$ of handling, rats underwent surgery. The rats were allowed at least $7 \mathrm{~d}$ to recover from surgery. Rats were then acclimated to the infusion procedure as described in Experiment 3.

Twenty-four hours after acclimation, the rats were conditioned in context A. On the conditioning day, the rats were transported to the conditioning chambers in squads of eight counterbalanced for each squad and group where they received auditory fear conditioning as described in Experiment 1. Twenty-four hours after conditioning, the rats underwent a context retention test. Before testing, squads of 8 rats were transported into the laboratory in individual white buckets for infusions of muscimol or saline as described in Experiment 3. After the infusion, $1 \mathrm{~min}$ was allowed for diffusion before the internal cannulas were removed. After the internal cannulas were removed, clean dummy cannulas were inserted into the guide cannulas, and the rats were immediately placed in the conditioning context where fear was assessed as described in Experiment 1. Seventy-two hours after conditioning animals were once again transported to the infusion room and infused as described above. Immediately after the infusion the rats were transported to a novel context where auditory fear was assessed as described in Experiment 1.

\section{Histology}

Histological verification of lesions and cannula placement location was performed after behavioral testing and completed as described in Experiment 1.

\section{Data Analysis}

Data analysis was preformed as described in Experiment 1.

\section{Acknowledgments}

This work was supported by a grant from NIMH (RO1MH073655) to S.M.

\section{References}

Anglada-Figueroa, D. and Quirk, G.J. 2005. Lesions of the basal amygdala block expression of conditioned fear but not extinction. I. Neurosci. 25: 9680-9685.

Balleine, B.W. and Killcross, S. 2006. Parallel incentive processing: An integrated view of amygdala function. Trends Neurosci. 29: 272-279.

Bevins, R.A., McPhee, J.E., Rauhut, A.S., and Ayres, J.J. 1997. Converging evidence for one-trial context fear conditioning with an immediate shock: Importance of shock potency. J. Exp. Psychol. Anim. Behav. Process. 23: 312-324.

Cahill, L., Vazdarjanova, A., and Setlow, B. 2000. The basolateral amygdala complex is involved with, but is not necessary for, rapid acquisition of Pavlovian 'fear conditioning.' Eur. J. Neurosci. 12: 3044-3050.

Campeau, S. and Davis, M. 1995. Involvement of the central nucleus and basolateral complex of the amygdala in fear conditioning measured with fear-potentiated startle in rats trained concurrently with auditory and visual conditioned stimuli. J. Neurosci. 15: 2301-2311.

Cousens, G. and Otto, T. 1998. Both pre- and posttraining excitotoxic lesions of the basolateral amygdala abolish the expression of olfactory and contextual fear conditioning. Behav. Neurosci. 112: $1092-1103$.

Davis, M. 1992. The role of the amygdala in fear and anxiety. Annu. Rev. Neurosci. 15: 353-375.

Davis, M. and Whalen, P.J. 2001. The amygdala: Vigilance and emotion. Mol. Psychiatry 6: 13-34.

Doron, N.N. and Ledoux, J.E. 2000. Cells in the posterior thalamus project to both amygdala and temporal cortex: A quantitative retrograde double-labeling study in the rat. J. Comp. Neurol. 425: 257-274.

Falls, W.A. and Davis, M. 1995. Lesions of the central nucleus of the amygdala block conditioned excitation, but not conditioned inhibition of fear as measured with the fear-potentiated startle effect. Behav. Neurosci. 109: 379-387.

Fanselow, M.S. 1990. Factors governing one-trial contextual conditioning. Anim. Learn. Behav. 18: 264-270.

Fendt, M. and Fanselow, M.S. 1999. The neuroanatomical and neurochemical basis of conditioned fear. Neurosci. Biobehav. Rev. 23: $743-760$.

Goosens, K.A. and Maren, S. 2001. Contextual and auditory fear conditioning are mediated by the lateral, basal, and central amygdaloid nuclei in rats. Learn. Mem. 8: 148-155.

Goosens, K.A. and Maren, S. 2003. Pretraining NMDA receptor blockade in the basolateral complex, but not the central nucleus, of the amygdala prevents savings of conditional fear. Behav. Neurosci. 117: $738-750$.

Goosens, K.A., Holt, W., and Maren, S. 2000. A role for amygdaloid PKA and PKC in the acquisition of long-term conditional fear memories in rats. Behav. Brain Res. 114: 145-152.

Helmstetter, F.J. 1992. The amygdala is essential for the expression of conditional hypoalgesia. Behav. Neurosci. 106: 518-528.

Holland, P.C. and Gallagher, M. 2003. Double dissociation of the effects of lesions of basolateral and central amygdala on conditioned stimulus-potentiated feeding and Pavlovian-instrumental transfer. Eur. J. Neurosci. 17: 1680-1694.

Killcross, S., Robbins, T.W., and Everitt, B.J. 1997. Different types of fear-conditioned behaviour mediated by separate nuclei within amygdala. Nature 388: 377-380.

Kim, M. and Davis, M. 1993. Electrolytic lesions of the amygdala block acquisition and expression of fear-potentiated startle even with extensive training but do not prevent reacquisition. Behav. Neurosci. 107: 580-595

Koo, J.W., Han, J.S., and Kim, J.J. 2004. Selective neurotoxic lesions of basolateral and central nuclei of the amygdala produce differential effects on fear conditioning. J. Neurosci. 24: 7654-7662.

LeDoux, J.E. 2000. Emotion circuits in the brain. Annu. Rev. Neurosci. 23: $155-184$.

LeDoux, J.E., Iwata, J., Cicchetti, P., and Reis, D.J. 1988. Different projections of the central amygdaloid nucleus mediate autonomic and behavioral correlates of conditioned fear. J. Neurosci. 8: $2517-2529$.

LeDoux, J.E., Cicchetti, P., Xagoraris, A., and Romanski, L.M. 1990. The lateral amygdaloid nucleus: Sensory interface of the amygdala in fear conditioning. J. Neurosci. 10: 1062-1069.

Lee, J. L., Dickinson, A., and Everitt, B. J. 2005. Conditioned suppression and freezing as measures of aversive Pavlovian conditioning: Effects of discrete amygdala lesions and overtraining. Behav. Brain Res. 159: 221-233.

Maren, S. 1998. Overtraining does not mitigate contextual fear conditioning deficits produced by neurotoxic lesions of the 
basolateral amygdala. J. Neurosci. 18: 3088-3097.

Maren, S. 1999. Neurotoxic basolateral amygdala lesions impair learning and memory but not the performance of conditional fear in rats. $J$. Neurosci. 19: 8696-8703.

Maren, S. 2001. Neurobiology of Pavlovian fear conditioning. Annu. Rev. Neurosci. 24: 897-931.

Maren, S. 2005a. Building and burying fear memories in the brain. Neuroscientist 11: 89-99.

Maren, S. 2005b. Synaptic mechanisms of associative memory in the amygdala. Neuron 47: 783-786.

Maren, S., Aharonov, G., and Fanselow, M.S. 1996. Retrograde abolition of conditional fear after excitotoxic lesions in the basolateral amygdala of rats: Absence of a temporal gradient. Behav. Neurosci. 110: $718-726$.

McDonald, A.J. 1998. Cortical pathways to the mammalian amygdala. Prog. Neurobiol. 55: 257-332.

Merino, S.M. and Maren, S. 2006. Hitting Ras where it counts: Ras antagonism in the basolateral amygdala inhibits long-term fear memory. Eur. J. Neurosci. 23: 196-204.
O'Reilly, R.C. and Rudy, J.W. 2001. Conjunctive representations in learning and memory: Principles of cortical and hippocampal function. Psychol. Rev. 108: 311-345.

Pare, D., Quirk, G.J., and Ledoux, J.E. 2004. New vistas on amygdala networks in conditioned fear. J. Neurophysiol. 92: 1-9.

Romanski, L.M. and LeDoux, J.E. 1992. Equipotentiality of thalamo-amygdala and thalamo-cortico-amygdala circuits in auditory fear conditioning. J. Neurosci. 12: 4501-4509.

Sanders, M.J., Wiltgen, B.J., and Fanselow, M.S. 2003. The place of the hippocampus in fear conditioning. Eur. J. Pharmacol. 463: 217-223.

Swanson, L. 1992. Brain maps: Structure of the rat brain. Elsevier, New York.

Wilensky, A.E., Schafe, G.E., Kristensen, M.P., and LeDoux, J.E. 2006. Rethinking the fear circuit: The central nucleus of the amygdala is required for the acquisition, consolidation, and expression of Pavlovian fear conditioning. J. Neurosci. 26: 12387-12396.

Received April 13, 2007; accepted in revised form July 11, 2007. 


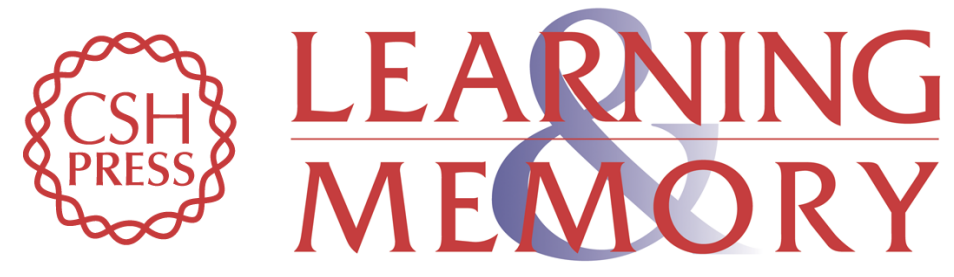

\section{The central nucleus of the amygdala is essential for acquiring and expressing conditional fear after overtraining}

Joshua M. Zimmerman, Christine A. Rabinak, Ian G. McLachlan, et al.

Learn. Mem. 2007, 14:

Access the most recent version at doi:10.1101//m.607207

References This article cites 37 articles, 10 of which can be accessed free at:

http://learnmem.cshlp.org/content/14/9/634.full.html\#ref-list-1

License

Email Alerting Receive free email alerts when new articles cite this article - sign up in the box at the Service top right corner of the article or click here. 